\title{
Direct Adaptive Compensation Control of Mechanical Systems With Unknown Actuator Failures and Dead-Zone Nonlinearities
}

\author{
Xiaohang Su and Zhi Liu ${ }^{\mathrm{a}}$ \\ School of Automation, Guangdong University of Technology, Guangzhou, China
}

\begin{abstract}
In this paper, a new tuning function backstepping control scheme is proposed for a class of parametric strict feedback nonlinear systems to accommodate actuator failures/faults and dead-zone constraints, where the failures/faults are uncertain in time, pattern, and values, and the dead-zone parameters are not available. Roughly speaking, such a scheme is developed in two steps below. First, by using an adaptive smooth inverse function to compensate for the dead-zone nonlinearity, we separate the coupling actuator dynamics into two parts, i.e., the dead-zone compensation errors and the nominal failure dynamics. Afterward, we further handle these two parts based on the techniques of robust adaptive approach and parametrization method. With our scheme, the global boundedness of the signals in the closed-loop system are ensured, and the tracking error is steered to zero asymptotically. These results have also been verified through simulation studies.
\end{abstract}

\section{Introduction}

In a practical control system, dead-zone characteristic ubiquitously exists in gear friction, hydraulic valves, DC motors and mechanical connection, which usually degrades the performance of the system. In recent years, adaptive compensation control schemes for dead-zone nonlinearity in the presence of parametric uncertainties have been developed. In [1], the dead-zone nonlinearity is regarded as an external bounded disturbance which can be mitigated through robust control design by using a direct decomposition method (DDM). Apart from DDM, the inverse compensation method (ICM) is another effective strategy to eliminate the effects of nonsmooth nonlinearities. Compared to the compensation method DDM, ICM can completely cancel actuator dead-zone by constructing an inverse compensator through parameter identification; see [2]. However, it is usually costly for offline parameter identification. Thus it is of significance to develop an adaptive inverse compensation method (AICM) by using the adaptive technique to estimate unknown dead-zone parameters online; see [3].

Besides the inherent actuator nonlinearities mentioned above, the failures/faults of suddenly getting stuck and losing partial effectiveness may also occur in practical actuation mechanisms. Recently, studies on actuator failures started by handling the linear system failure in [4], and the

${ }^{\mathrm{a} C}$ Corresponding author : lz@gdut.edu.cn 
results were further extended to nonlinear system failure in [5] by the utilizing backstepping technique. With backstepping recursive design, a new prescribed performance bounds (PPB) based controller is proposed in [6] to guarantee the tracking error within the prescribed bounds. Moreover, in the recent work [7], a robust adaptive fault-tolerant control scheme is further proposed for compensating failures in dead-zone actuators. However, such a scheme mainly treats the actuator failures and dead-zone nonlinearity as bounded disturbance-like effects, and thus the perfect asymptotic tracking performance cannot be obtained even for the case of finite number of actuator failures.

Motivated by observations above, in this paper, we study the problem of direct adaptive failure compensation control for a class of uncertain multiple inputs and single output (MISO) nonlinear systems with actuator failures and dead-zone constraints. Such problem is successfully addressed with our newly proposed control methodology with the following contributions.

1) Note that we take actuator failures and dead-zone nonlinearities into account simultaneously in control design, which thus is more general than the existing work [8] that only considered dead-zone effect and [5] that solely focused on actuator failure compensation. Moreover, perfect asymptotic tracking control performance is achieved, irrespective of the existence of such both adversaries.

2) The smooth adaptive inverse is firstly utilized to compensate for dead-zone nonlinearity, such that the compensating errors containing failure parameters between actual function value of dead-zone nonlinearity and its designed dead-zone output, which is then eliminated in the subsequent design.

3) Recently, the authors in [7] proposed a new and novel robust adaptive control scheme, which has been shown to be applicable to compensate for actuator dead-zone and failures/faults. However, such a scheme cannot recover the perfect asymptotic tracking performance when the number of actuator failures become finite. This issue is also successfully addressed with our proposed scheme.

The outline of the paper is organized below. In Section 2, the control problem is formulated, and the related assumptions are given. In Section 3, a desired controller for addressing control problems is constructed and the stability analysis is provided at the end of this section. Finally, simulations on mechanical system with dead-zone and failures/faults are shown in Section 4.

\section{System model and problem statement}

In this paper, the following uncertain nonlinear system for the adaptive actuator failure compensation problem is considered.

$$
\begin{aligned}
& \dot{x}_{i}=x_{i+1}+\varphi_{i}^{T}\left(x_{1}, x_{2}, \ldots, x_{i}\right) \vartheta, i=1,2, \ldots, \lambda-1 \\
& \dot{x}_{\lambda}=\varphi_{0}(x, \eta)+\varphi_{\lambda}^{T}(x, \eta) \vartheta+\sum_{j=1}^{q} b_{j} \gamma_{j}(x, \eta) u_{j} \\
& \mathrm{y}=x_{1}
\end{aligned}
$$

where $x_{i} \in \mathfrak{R}^{i}$ are state variables, $y \in \mathfrak{R}$ is the system output, $u_{j} \in \mathfrak{R}$ for $j=1,2, \ldots, q$ denotes the $j$ th control input to the plant, $\varphi_{0} \in \mathfrak{R}, \varphi_{i}(x) \in \mathfrak{R}^{p}(i=1,2, \ldots, \lambda)$ and $\gamma_{j}(x, \eta) \neq 0(j=1,2, \ldots, q)$ are known smooth nonlinear functions, $\vartheta \in \mathfrak{R}^{p}$ and $b_{j}(j=1,2, \ldots, q)$ are unknown parameters and control coefficients, respectively. Denote $\tau_{j}$ as the input of the $j$ th actuator $(j=1,2, \ldots, q), u_{j}$ as the output of the $j$ th actuator $(j=1,2, \ldots, q)$. An actuator with its input equals to its output, i.e., $\tau_{j}=u_{j}$, namely the failure-free actuators.

As previous analysis, dead-zone is inevitable in most mechanical devices, which can be denoted as 


$$
v_{j}=D\left(\tau_{j}\right)= \begin{cases}m_{r}\left(\tau_{j}-d_{+}\right) & \tau_{\mathrm{j}} \geq \mathrm{d}_{+} \\ 0 & \mathrm{~d}_{-}<\tau_{\mathrm{j}}<\mathrm{d}_{+} \\ m_{l}\left(\tau_{j}-d_{-}\right) & \tau_{\mathrm{j}} \leq \mathrm{d}_{-}\end{cases}
$$

where $v_{j}$ for $j=1,2, \ldots, q$ is the $j$ th output of the dead-zone actuator. $d_{-} \leq 0, d_{+} \geq 0$ and $m_{r}>0, m_{l}>0$ are unknown constants.

Considering the dead-zone actuator with possible unknown failures/faults, the effect of failure nonlinearity can not be simply taken as an external disturbance-like term without considering its structural information, thus the mathematic model for failure is needed below:

$$
\begin{gathered}
u_{j}=\rho_{j} v_{j}+v_{s j}, \forall t \geq t_{j F} \\
\rho_{j} v_{s j}=0, j=1,2, \ldots q
\end{gathered}
$$

where $\rho_{j} \in[0,1), v_{s j}$ and $t_{j F}$ are all unknown constants.

The control objectives of this paper are to ensure all the signals of the nonlinear system bounded and the system output $y$ asymptotically tracks the reference signal $y_{r}$. To achieve the objectives, the following assumptions are needed.

Assumption 1. The dead-zone parameters satisfy $m_{r} \geq m_{r 0}, m_{l} \geq m_{l 0}$, where $m_{r 0}, m_{l 0}$ are two small positive constants, as described in [8].

Assumption 2. The reference signal $y_{r}$ and its first $\lambda$ th order derivatives $y_{r}^{(i)}(i=1,2, \ldots, \lambda)$ are known, bounded and piecewise continuous. $\gamma_{i}(x, \eta) \neq 0$, and the signs of $b_{i}$, i.e., $\operatorname{sgn}\left(b_{i}\right)$ are known.

\section{Objective controller design for solving the problem}

\subsection{Dead-zone Inverse Model}

In this part, to prevent the system from potential chattering phenomenon in the backstepping recursion, $v_{j}$ needs to be designed as

$$
v_{d j}=-\hat{\theta}_{j}^{T} \hat{w}_{j}, j=1,2, \ldots, q
$$

where $\quad \hat{\theta}_{j}^{T}=\left[m_{r}, m_{r} d_{+}, m_{l}, m_{l} d_{-}\right] \quad$ is $\quad$ an estimate of $\theta_{j}$, $\hat{w}_{j}^{T}=\left[-\Phi_{1}(\tau) \tau_{j}, \Phi_{1}(\tau),-\Phi_{2}(\tau) \tau_{j},-\Phi_{2}(\tau)\right]$ is an identity matrix. $\Phi_{1}\left(v_{j}\right)=\frac{e^{\frac{v_{j}}{e_{0}}}}{e^{\frac{-v_{j}}{e_{0}}}+e^{\frac{v_{j}}{e_{0}}}}$ and $\Phi_{2}\left(v_{j}\right)=\frac{e^{-\frac{v_{j}}{e_{0}}}}{e^{\frac{-v_{j}}{e_{0}}}+e^{\frac{v_{j}}{e_{0}}}}$ are designed as smooth continuous functions, where $e_{0}>0$ is a user-defined parameter.

Then the corresponding actuator smooth dead-zone inverse model is obtained as: 


$$
\tau_{j}=\frac{v_{d j}+m_{r} d_{+}}{\hat{m}_{r}} \Phi_{1}\left(v_{d j}\right)+\frac{v_{d j}+m_{l} d_{-}}{\hat{m}_{l}} \Phi_{2}\left(v_{d j}\right)
$$

The compensating errors between $v_{j}$ and $v_{d j}$ are obtained as

$$
v_{j}-v_{d j}=-\tilde{\theta}_{j}^{T} \hat{w}_{j}+D_{N j}
$$

where $\tilde{\theta}_{j}=\theta_{j}-\hat{\theta}_{j}, D_{N j}=\theta_{j}^{T}\left(\hat{w}_{j}-w_{j}\right) . D_{N j}$ can be guaranteed to be bounded for all $t \geq 0$, see, $[8]$.

\subsection{Controller Design}

In this subsection, the novel adaptive compensation scheme for actuator failures and dead-zone constraints of nonlinear system is proposed. First of all, the following error variables are introduced as

$$
\begin{gathered}
z_{1}=x_{1}-y_{r} \\
z_{i}=x_{i}-\alpha_{i-1}-y_{r}^{(i-1)}, i=2,3, \ldots, \lambda
\end{gathered}
$$

Step $\mathbf{i}(i=1,2, \ldots, \lambda-1)$. Based on the tuning function design scheme, the $i$ th stabilizing function $\alpha_{i}$ and tuning function $\varsigma_{i}$ are chosen as

$$
\begin{gathered}
\alpha_{1}=-c_{1} z_{1}-\varpi_{1}^{T} \hat{\vartheta} \\
\alpha_{i}=-z_{i-1}-c_{i} z_{i}-\varpi_{i}^{T} \hat{\vartheta}+\frac{\partial \alpha_{i-1}}{\partial \hat{\vartheta}} \Gamma \varsigma_{i}+\sum_{j=1}^{i-1}\left[\frac{\partial \alpha_{i-1}}{\partial x_{j}} x_{j+1}+\frac{\partial \alpha_{i-1}}{\partial y_{r}^{(j-1)}} y_{r}^{(j)}\right]+\sum_{j=2}^{i-1} \frac{\partial \alpha_{j-1}}{\partial \hat{\vartheta}} \Gamma \varpi_{i} z_{j}, i=2, \ldots, \lambda-1 \\
\varsigma_{i}=\varsigma_{i-1}+\varpi_{i} z_{i}, \varpi_{i}=\varphi_{i}-\sum_{j=1}^{i-1} \frac{\partial \alpha_{i-1}}{\partial x_{j}} \varphi_{j}
\end{gathered}
$$

Step $\lambda$. From the above definitions, $\dot{z}_{\lambda}$ is obtained as

$$
\dot{z}_{\lambda}=\varphi_{0}+\varphi_{\lambda}^{T} \vartheta-\dot{\alpha}_{\lambda-1}-y_{r}^{(\lambda)}-\sum_{i=1}^{q} b_{i} \gamma_{i}(x, \eta) \rho_{i} \tilde{\theta}_{i}^{T} \hat{w}_{i}+\sum_{i=1}^{q} b_{i} \gamma_{i} \rho_{i} D_{N i}+\sum_{i=1}^{q} b_{i} \gamma_{i}\left(\rho_{i} v_{d i}+v_{s i}\right)
$$

Remark 1: Based on the adaptive inverse compensation method (ACIM), the compensating errors between actual function value and its designed dead-zone output are obtained, as seen in (7). By combining the failure model (3)-(4), we can separate the coupling actuator dynamics into two parts: the dead-zone compensation errors containing failure parameters and the nominal failure dynamics, as shown in (13). These two parts can be compensated by synthesizing robust control approach, parametrization method and direct adaptive approach in following steps.

Supposing one or more of failures suddenly occur in the dead-zone actuators during time interval $\left[T_{K-1}, T_{K}\right)$, where $T_{K-1}, T_{k}$ for $k=1,2, \ldots, e, e+1(e \leq q)$ are a finite number of time instants. We define two sets such that $Q_{\text {tot }_{k}}=\left\{j_{1,1}, j_{1,2}, \ldots, j_{1, q_{t o t_{k}}}\right\}$ and $Q_{\text {par }_{k}}=\left\{j_{1,1}, j_{1,2}, \ldots, j_{1, q_{p a u_{k}}}\right\}$. As previous analysis, the unknown parameters $D_{N i}$ in (13) can be computed as 


$$
\left|b_{i} D_{N i}\right| \leq\left|b_{i}\right| \bar{D}_{N i}=\left|b_{i}\right|\left(\tilde{\bar{D}}_{N i}+\hat{\bar{D}}_{N i}\right)
$$

Then the Lyapunov function $V_{\lambda}$ is chosen as

$$
V_{\lambda}=V_{\lambda-1}+\frac{1}{2} z_{\lambda}^{2}+\sum_{i=1, i \in Q_{\text {lotk }}}^{q} \frac{\rho_{i}\left|b_{i}\right|}{2} \tilde{\kappa}^{T} \Gamma_{k}^{-1} \tilde{\kappa}+\sum_{i=1}^{q} \frac{\rho_{i}\left|b_{i}\right|}{2} \tilde{\theta}^{T} \Gamma_{s}^{-1} \tilde{\theta}+\sum_{i=1}^{q} \frac{\rho_{i}\left|b_{i}\right|}{2} \tilde{\bar{D}}_{N i}^{2}
$$

where $\Gamma_{k}$ and $\Gamma_{s}$ are two positive-definite matrices. If $b_{i}, \rho_{i}$ and $v_{s i}$ for $i=1,2, \ldots, q, h \in Q_{\text {tot }_{k}}$ are known, $\kappa$ is a desired constant vector such that

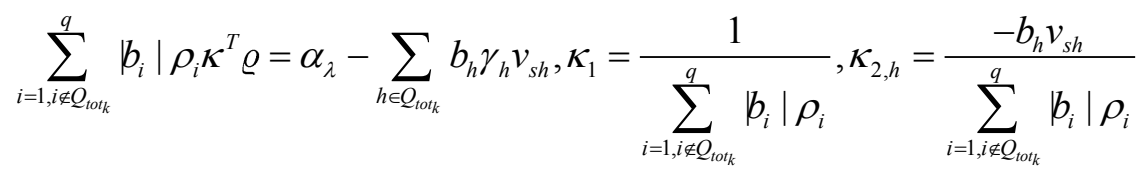

where $h \in Q_{\text {tot }_{k}}$ and $h \in\{1,2, \ldots, q\} \Rightarrow \kappa_{2, h}=0, \hat{\kappa}=\left[\hat{\kappa}_{1}, \hat{\kappa}_{2}^{T}\right]^{T}, \hat{\kappa}_{2}=\left[\hat{\kappa}_{2,1}, \hat{\kappa}_{2,2}, \ldots, \hat{\kappa}_{2, q}\right]^{T}$, $\varrho=\left[\alpha_{\lambda}, \gamma_{1}, \ldots, \gamma_{q}\right]^{T}$. Then $\dot{V}_{\lambda}$ can be computed as

$$
\begin{aligned}
& \dot{V}_{\lambda} \leq-\sum_{i=1}^{\lambda-1} c_{i} z_{i}^{2}+z_{\lambda}\left\{z_{\lambda-1}+\alpha_{\lambda}+\varphi_{0}+\varpi_{\lambda}^{T} \hat{\vartheta}-\sum_{i=1}^{\lambda-1}\left[\frac{\partial \alpha_{\lambda-1}}{\partial x_{i}} x_{i+1}+\frac{\partial \alpha_{\lambda-1}}{\partial y_{r}^{(i-1)}} y_{r}^{(i)}\right] n-\frac{\partial \alpha_{\lambda-1}}{\partial \hat{\vartheta}} \dot{\hat{\vartheta}}\right. \\
& \left.+\sum_{i=1}^{q} b_{i} \gamma_{i}(x, \eta)\left(\rho_{i} v_{d i}+v_{s i}\right)+\sum_{i=1}^{q} b_{i} \mid \rho_{i} \gamma_{i} \hat{\bar{D}}_{N i}-y_{r}^{(\lambda)}\right\}+\tilde{\vartheta}^{T}\left(\varsigma_{\lambda}-\Gamma^{-1} \dot{\hat{\vartheta}}\right)+\sum_{i=2}^{\lambda-1} z_{i} \frac{\partial \alpha_{i-1}}{\partial \hat{\vartheta}}\left(\Gamma \varsigma_{\lambda-1}-\dot{\hat{\vartheta}}\right) \\
& \left.\left.-\sum_{i=1, i \notin Q_{\text {oto }}}^{q} b_{i} \mid \rho_{i} \widehat{\kappa_{i}^{T}\left(\varrho z_{\lambda}\right.}+\Gamma_{k}^{-1} \dot{\hat{\kappa}}\right)+\sum_{i=1}^{q} b_{i}\left|\rho_{i} \tilde{\theta}_{i}^{T}\left[\left|\gamma_{i} \hat{w}_{i} z_{\lambda}\right|-\Gamma_{s}^{-1} \dot{\hat{\theta}}_{i}\right]+\sum_{i=1}^{q}\right| b_{i}\left|\rho_{i} \widetilde{\bar{D}_{N i}\left[\mid \gamma_{i}\right.} z_{\lambda}\right|-\dot{\hat{\bar{D}}}_{N i}\right]
\end{aligned}
$$

To make $\dot{V}_{\lambda}$ non-positive, the tuning function $\varsigma_{\lambda}$ and the stabilizing function $\alpha_{\lambda}$ are defined as

$$
\begin{gathered}
\varsigma_{\lambda}=\varsigma_{\lambda-1}+\varpi_{\lambda} z_{\lambda}, \varpi_{\lambda}=\varphi_{\lambda}-\sum_{j=1}^{\lambda-1} \frac{\partial \alpha_{\lambda-1}}{\partial x_{j}} \varphi_{j} \\
\alpha_{\lambda}=-z_{\lambda-1}-c_{\lambda} z_{\lambda}-\varphi_{0}-\varpi_{\lambda}^{T} \hat{\vartheta}+\frac{\partial \alpha_{\lambda-1}}{\partial \hat{\vartheta}} \Gamma \varsigma_{\lambda}+\sum_{i=2}^{\lambda-1} \frac{\partial \alpha_{i-1}}{\partial \hat{\vartheta}} \Gamma \varpi_{\lambda} z_{i} \\
+\sum_{i=1}^{\lambda-1}\left(\frac{\partial \alpha_{\lambda-1}}{\partial x_{i}} x_{i+1}+\frac{\partial \alpha_{\lambda-1}}{\partial y_{r}^{(i-1)}} y_{r}^{(i)}\right)-\sum_{i=1}^{q} b_{i} \mid \rho_{i} \gamma_{i} \hat{\bar{D}}_{N i}+y_{r}^{(\lambda)}
\end{gathered}
$$

The final parameter update laws are chosen as

$$
\dot{\hat{\vartheta}}=\Gamma \varsigma_{\lambda}, \dot{\hat{\kappa}}=-\Gamma_{k} \varrho z_{\lambda}, \dot{\hat{\theta}}_{i}=\Gamma_{s}\left|\gamma_{i} \hat{w}_{i} z_{\lambda}\right|, \dot{\bar{D}}_{N i}=\left|\gamma_{i} z_{\lambda}\right|
$$

The control law $v_{d i}$ is finally determined as 


$$
v_{d i}=\operatorname{sgn}\left(b_{i}\right) \frac{1}{\gamma_{i}} \hat{\kappa}^{T} \varrho, \text { for } i=1,2, \ldots, q
$$

\subsection{Stability Analysis}

Theorem: Considering the closed-loop adaptive system consists of the plant (1) with the control law (21) and the parameter update laws (20) in the presence of dead-zone nonlinearity (2) and actuator failures (3)-(4) and under Assumptions 1-2. The boundedness of all the signals are ensured and the tracking error approaches to zero asymptotically, i.e., $\lim _{t \rightarrow \infty}\left[y(t)-y_{r}(t)\right]=0$.

Proof. From Step i, we conclude that

$$
\begin{gathered}
\dot{z}_{1}=z_{2}-c_{1} z_{1}+\varpi_{1}^{T} \tilde{\vartheta} \\
\dot{z}_{i}=z_{i+1}-c_{i} z_{i}-z_{i-1}+\varpi_{i}^{T} \tilde{\vartheta}+\frac{\partial \alpha_{i-1}}{\partial \hat{\vartheta}}\left(\Gamma \varsigma_{i}-\dot{\hat{\vartheta}}\right)+\sum_{j=2}^{i-1} \frac{\partial \alpha_{j-1}}{\partial \hat{\vartheta}} \varpi_{i} z_{j}
\end{gathered}
$$

Among $\left[0, T_{1}\right)$, with the control law (20), supposing that $b_{i}$ is known and $\kappa_{2, h}=0$ for $k=1,2, \ldots, q$ is chosen, the derivative of $z_{\lambda}$ then satisfies

$$
\dot{z}_{\lambda}=-c_{\lambda} z_{\lambda}-z_{\lambda-1}+\varpi_{\lambda}^{T} \tilde{\vartheta}-\sum_{i=1}^{q} b_{i} \mid \tilde{\kappa}^{T} \varrho+\sum_{j=2}^{i-1} \frac{\partial \alpha_{j-1}}{\partial \hat{\vartheta}} \varpi_{i} z_{j}-\sum_{i=1}^{q} b_{i} \gamma_{i}(x, \eta) \rho_{i}\left(\tilde{\theta}_{i}^{T} \hat{w}_{i}-D_{N i}\right)
$$

Assuming during the time interval $\left[T_{k-1}, T_{k}\right)$ for $k=2,3, \ldots, r$, the system with the controller (21) and update laws (20) undergoes dead-zone nonlinearity and PLOE type of failure, then we have

$$
\dot{V}_{k-1} \leq-\sum_{j=1}^{\lambda} c_{j} z_{j}^{2}
$$

where $V_{k-1}\left(T_{k}+\Delta(t)\right) \leq V_{k-1}\left(T_{k}\right)$, which shows that $z(t), \tilde{\vartheta}, \tilde{\kappa}, \tilde{\theta}, \tilde{D}_{N} \in L_{\infty}$, then the boundedness of $x_{1}(t), \ldots, x_{\lambda}(t)$ can be derived successively. Besides, by using Barbalat's lemma and combining with $\dot{z}(t) \in L_{2}$, the error vector $z(t)$ asymptotic convergence to zero can be proved, i.e., $\lim _{t \rightarrow \infty} z(t)=0$. The proof of the theorem is completed.

\section{Simulation studies}

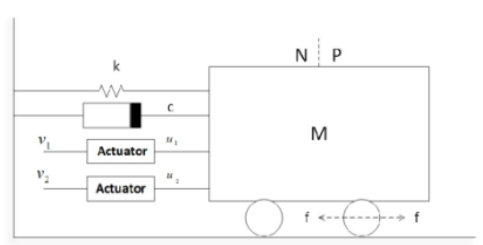

Figure 1. Spring-mass-damper control system with dual actuators. 


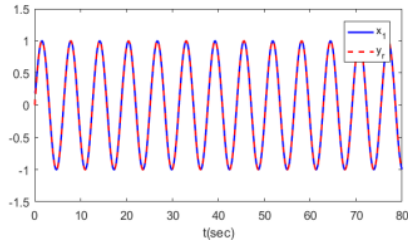

Figure 2

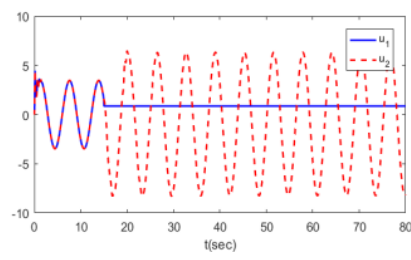

Figure 3

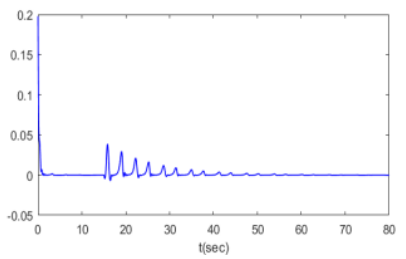

Figure 4

In this example, we apply our approach to the following physical system with dual actuators. For the, visualization, the corresponding physical model is designed in Fig. 1. In addition, the dynamic mathematic model is given as

$$
\begin{aligned}
& \dot{x}_{1}=x_{2} \\
& \dot{x}_{2}=-\frac{k}{M} x_{1}-\frac{c}{M} x_{2}+\frac{1}{M} \sum_{i=1}^{2} u_{i}
\end{aligned}
$$

where $y=x_{1}$ is regarded as the output position of the car whose mass is denoted by $M=1 \mathrm{~kg}$. $k=8 N / \mathrm{s}$ is the stiffness of the spring, and $c=2 N \cdot m / s$ represents the damping coefficient. For simulation, the initial values are given as $x_{1}(0)=0.1 m, x_{2}(0)=0 m / s, u_{1}(0)=u_{2}(0)=0 N$ and the reference signal is $y_{r}=\sin (t)$. The dual actuator failure terms are modeled as: at 15 th second, the actuator $u_{1}$ suffers partial loss of effectiveness (PLOE) as $u_{1}=70 \% v_{1}$. Besides, the maximum static friction of this physical model is chosen as $1 N$, which can be regarded as a dead-zone nonlinearity whose parameters are defined as $d_{+}=1 N, d_{-}=-1 N, m_{r}=1, m_{l}=1$.

By choosing the other simulation parameters as $c_{1}=3, c_{2}=2.8$, the results are shown in Fig. 2Fig. 4. As presented in Fig. 3, the actuator $u_{1}$ suffers failure and actuator $u_{2}$ works in good condition. By using our proposed control scheme, it can be seen from Fig. 2 that the output signal $x_{1}$ tracks the reference signal $y_{r}$ perfectly. Besides in Fig. 4, the tracking error asymptotically approaches to zero.

\section{Acknowledgments}

This work was supported in part by the National Natural Science Foundation of China under Grant 61573108, in part by National Natural Science Foundation of China (U1501251), in part by the Natural Science Foundation of Guangdong Province 2016A030313715, in part by the Natural Science Foundation of Guangdong Province through the Science Fund for Distinguished Young Scholars under Grant S20120011437, in part by the Ministry of Education of New Century Excellent Talent under Grant NCET-12-0637.

\section{References}

1. Z. Li and C. Y. Su. Neural-adaptive control of single-master-multiple-slaves teleoperation for coordinated multiple mobile manipulators with time-varying communication delays and input uncertainties. IEEE Transactions on Neural Networks and Learning Systems, 24(9):1400-1413, September 2013. 
2. D. A. Recker, P. V. Kokotovic, D. Rhode, and J. Winkelman. Adaptive nonlinear control of systems containing a deadzone. Proceedings of the 30th IEEE Conference on Decision and Control, 1991, pages 2111 - 2115, December 1991.

3. G. Tao and P. V. Kokotovic. Adaptive sliding control of plants with unknown dead-zone. IEEE Trans. Autom. Control, 39:59 - 68, January 1994.

4. G. Tao, S. Chen, and S. M. Joshi. An adaptive actuator failure compensation controller using output feedback. IEEE Transactions on Automatic Control, 47(3):506 - 511, March 2002.

5. X. D Tang, G. Tao, and S. M. Joshi. Adaptive actuator failure compensation for nonlinear MIMO systems with an aircraft control application. Automatica, 43(11):1869 - 1883, November 2007.

6. W. Wang and C. Y Wen. Adaptive actuator failure compensation control of uncertain nonlinear systems with guaranteed transient performance. Automatica, 46(12):2082 - 2091, December 2010.

7. Y. X. Li and G. H. Yang. Adaptive fuzzy decentralized control for a class of large-scale nonlinear systems with actuator faults and unknown dead zones. IEEE Transactions on Systems, Man, and Cybernetics: Systems, PP(99):1 - 12, 2016.

8. J. Zhou, C. Wen, and Y. Zhang. Adaptive output control of nonlinear systems with uncertain dead-zone nonlinearity. IEEE Transactions on Automatic Control, 51(3):504 - 511, March 2006. 